\title{
A New Approach Based on Artificial Intelligent Models for Forecasting of Financial Markets
}

\author{
Ali Ghezelbash ${ }^{1 *}$, Farshid Keynia ${ }^{2}$ \\ ${ }^{*}$ Corresponding author, Lecturer, Faculty of Engineering, University of Jiroft, Jiroft, Iran. \\ E-mail: teacher.ghezelbash@yahoo.com \\ ${ }^{2}$ Energy Department, International Center for Science, High Technology \& Environmental Sciences, Kerman, \\ Iran.E-mail: keynia@yahoo.com
}

\begin{abstract}
This paper presents a study of artificial neural networks for use in stock price prediction. The goal of the article is to research financial market efficiency and to recognize major reversal points of long-term trend of stock market index, which could indicate a forthcoming crisis or market raise periods. Software was developed by using MATLAB to simulate the performance and efficiency of the algorithm.

A multilayer perception (M.L.P) neural network model is used to determine and explore the relationship between some variables as independent factors and the level of stock price index as a dependent element in the stock market under study over time. The results show that the neural network models can get better outcomes compared with parametric models like regression and others traditional statistical techniques. The hybridized approach was tested with published stock data and the results obtained showed remarkable improvement over the use of only technical analysis variables. Also, the prediction from hybridized approach was found satisfactorily adequate as a guide for traders and investors in making qualitative decisions.
\end{abstract}

Keywords: Stock Price Prediction, Back Propagation, Neural Network Architecture

\section{INTRODUCTION}

The stock market is one of the most popular investments, owing to its high expected profit. However, the higher the expected profit, the higher the implied risk. The stock market, which has been investigated by various studies, is a rather complicated environment. Neural networks have attracted increasing attention from researchers in many fields, including information processing, computer science, economics, medicine and mathematics, and have been used to solve a wide range of problems such as data mining, function approximation, pattern recognition, expert system and data prediction, etc. The widespread popularity of ANNs in many fields is mainly due to their ability to approximate complex multivariate nonlinear functions directly from the input samples. Neural networks can provide models for a large class of natural and artificial phenomena that are difficult to handle using classical parametric techniques [1].

Accurate prediction of stock prices presents a challenging task for traders and investors. Multitude of economical, social, political and psychological factors interacts in a complex way to form stock movement patterns. The early Efficient Market Theory (EMT) claims that prices move in a random way and it is not possible to develop an algorithm of some kind that predicts stock prices [2]. Other researchers contradicted this claim and presented considerable evidence showing that stock prices are, to some extent, predictable. Forecasting or predicting stock prices may be done following one or a combination of four approaches: fundamental analysis approach, technical analysis approach, time series forecasting and machine learning $[3,4,5]$.

In the early1900s Bachelier indicated similarities of stock price changes to the Brownian motion, or the stochastic process. Such results directed Fama [6] to formulate the efficient market hypothesis (EMH), which states that all information, which could affect stock prices is already incorporated into them, therefore the best guess of stock prices has to be equal to the current price. The validity of the efficient market hypothesis is questioned in numerous research works. Some of the researchers explore exceptions of the efficient market hypotheses, which occur due to regular or rare events, such as crises, seasonal impacts, market news, calendar anomalies (day-of-the week, holiday and other phenomena), or specific features of the particular financial markets according to their maturity [7].

The issue of stock price forecasting is analyzed by various methods of soft computing, including ANNs, probabilistic, and hybrid methods [8]. The scientific research of applying neural networks, hybrid and other intelligent analysis methods mostly use the financial variables of market index and return as model input, reflecting continuous changes of financial market time series. One of the indicators of analysis of financial 
series over the selected period is information efficiency, related to measurement of long term correlation memory [8].

Garcia and Gencay (2000), Gencay (1998), and Qi and Madala (1999) employ ANNs in stock market predictions. Qi and $\mathrm{Wu}$ (2003), who employ an ANN model with monetary fundamentals, find that their model cannot surpass the random walk model. Alternatively, Kiani (2005) and Kiani et al. (2005) use ANN models with macroeconomic time series and find that these outperform the linear as well as other nonlinear models employed. O'Connor and Madden (2005) evaluate the effectiveness of using ANNs with external indicators, such as commodity prices and currency exchange rates, in predicting movements in the Dow Jones Industrial Average index. Their results show that there are a few benefits to using these indicators over traditional methods based on historical data output only [9].

The focus of this paper is to improve the accuracy of stock price prediction by using the hybrid approach that combines the variables of technical and fundamental analysis for the creation of neural network predictive model for stock price prediction. The technical analysis variables are the core stock market indices (current stock price, opening price, closing price, volume, highest price and lowest price, etc.) while the fundamental analysis variables are company performance indices (price per annual earning, rumor/news, book value and financial status etc.).

\section{THE METHODOLOGY FOR EVALUATION OF MAIN INDEXES}

Numerous investigations have given rise to different decision support systems for the sake of providing investors with optional predictions. For a long time, many stock market experts have employed technical analysis for better predictions. Generally speaking, a technical analysis derives a stock's movement from the stock's own historical value. The historical data can be used directly to form support and resistance levels, or they can be plugged into many technical indicators for further investigation. Conventional research addressing this problem has generally employed time series analysis techniques that is, mixed auto regression moving average (ARMA) methods as well as multiple regression models [10]. Considerable evidence exists that shows that the stock market price is to some extent predictable [11].

Consider the basic structure of a feed-forward BPN with a single hidden layer as shown in Fig 1[12]. The network consists of $N$ input nodes, $K$ has hidden nodes and $M$ output nodes. Let $o_{p m}$ and $\bar{o}_{p m}$ be the output of output node $m$ and hidden node $k$ from input pattern $p$, respectively. Assume $\omega_{\mathrm{km}}$ is the network weight for hidden node $k$ and output node $m$, and $\bar{\omega}_{\text {nk }}$ is the network weight for input node $n$ and hidden node $k$. Also, let $x_{\mathrm{pn}}$ be the input value in input node $n$ for input pattern $p$, and $t_{\mathrm{pm}}$ be the target output value in the output node $m$ for input pattern $p$. Note that the symbol $\Delta$ represents the difference between the current and the new value in the next iteration. The standard BP algorithm is shown below:

(A) Initialization: Initialize all weights and refer to them as current weight $\omega_{\mathrm{km}}(0)$ and $\bar{\omega}_{\mathrm{nk}}(0)$. Set the learning rate $\mathrm{m}$ and the momentum factor $\alpha$ to small positive values (e.g. 0.1). Set the error threshold $E$ and the iteration number $i=0$.

(B) Forward Pass: Select the input pattern $x_{\mathrm{p}}=\left\{\mathrm{x}_{\mathrm{p} 1}, \ldots, \mathrm{x}_{\mathrm{pN}}\right\}$ from the training set and compute $o_{p m}(i)$ and $\bar{o}_{k m}(i)$ using the following equations [13]:

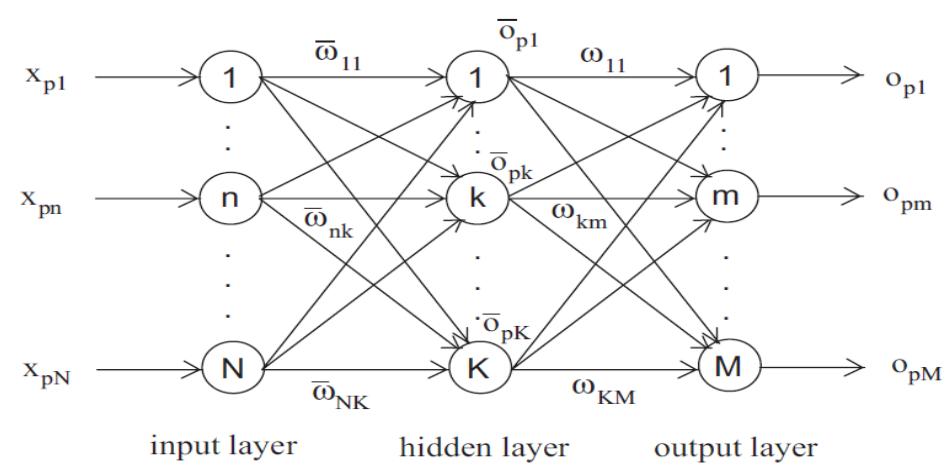

Fig 1: Basic structure of a feedforward BPN 


$$
\begin{aligned}
& o_{p m}(i)=f\left(\sum^{k} \omega_{k m}(i) \bar{o}_{p k}(i)\right) \\
& \bar{o}_{p k}(i)=f\left(\sum_{k=1}^{k} \bar{\omega}_{n k}(i) x_{p n}(i)\right)
\end{aligned}
$$

Where the activation function is $f(x)=1 /\left(1+e^{-x}\right)$.

Use the desired target $t_{p}=\left\{t_{p l}, \ldots, t_{p M}\right\}$ associated with xp to compute the sum of the squared system error, $E(i)$, for all input patterns as follows [14]:

$$
E(i)=\frac{1}{2} \sum_{p=1}^{p} \sum_{m=1}^{M}\left[t_{p m}-o_{p m}(i)\right]^{2}
$$

If $E(i) \leq E$, then the algorithm is completed and the convergence is achieved. Otherwise, go to step (Backward Pass).

(C) Backward Pass: Compute the changes of the weights for the next iteration $\Delta \omega_{k m}(i+1)$ and $\Delta \bar{\omega}_{n k}(i+1)$ using the following equations where $\mu$ is the learning rate and $\alpha$ is the momentum [13]:

$$
\begin{aligned}
& \omega_{k m}(i+1)=-\mu \frac{\partial E(i)}{\partial \omega_{k m}(i)}+\alpha \Delta \omega_{k m}(i)=\mu \sum_{p=1}^{p} \delta_{p m}(i) \bar{o}_{p k}(i)+\alpha \Delta \omega_{k m}(i) \\
& \Delta \bar{\omega}_{n k}(i+1)=-\mu \frac{\partial E(i)}{\partial \bar{\omega}_{n k}(i)}+\alpha \Delta \bar{\omega}_{n k}(i)=\mu \sum_{p=1}^{p} \bar{\delta}_{p k}(i) x_{p n}(i)+\alpha \Delta \bar{\omega}_{n k}(i)
\end{aligned}
$$

Where

$$
\begin{aligned}
& \delta_{p m}=\left(t_{p m}-o_{p m}(i)\right) o_{p m}(i)\left(1-o_{p m}(i)\right) \\
& \bar{\delta}_{p k}=\bar{o}_{p k}(i)\left(1-\bar{o}_{p k}(i)\right) \sum_{m=1}^{M} \delta_{p m}(i) \omega_{k m}(i)
\end{aligned}
$$

Update the weights for the next iteration $\Delta \omega_{k m}(i+1)$ and $\Delta \bar{\omega}_{k m}(i+1)$ and by considering the equations [15]:

$$
\begin{gathered}
\omega_{k m}(i+1)=\omega_{k m}(i)+\Delta \omega_{k m}(i+1) \\
\bar{\omega}_{k m}(i+1)=\bar{\omega}_{k m}(i)+\Delta \bar{\omega}_{k m}(i+1)
\end{gathered}
$$

Due to its relative simplicity, the BPN has become the most widely used NN in recent years. There are further issues when using the BPN, including the appropriate number of hidden nodes and layers, selecting the training data, fine tuning the learning rate and momentum, avoiding overtraining, the use of threshold, and so on. 


\section{APPLICATIONS IN STOCK MARKET INDEX FORECASTING}

The objective of this research work is to improve the accuracy of daily stock price prediction of stock market indices using artificial neural networks. The study used three-layer (one hidden layer) multilayer perceptron models (a feedforward neural network model) trained with backpropagation algorithm. Historical stock prices of different companies were obtained from published stock data on the Internet. The learning function or the activation function that was used is sigmoid function. Generally, the algorithm for our ANN experiment is shown in Fig 2 below [16, 17, 18].

(1) Define the output

(2) Choose the appropriate network architecture and algorithm. Multi-layer

perceptron model trained with backpropagation algorithm was primarily chosen.

(3) Determine the input data and preprocess if necessary.

(4) Choose appropriate learning function.

(5) Choose the appropriate network structure.

(6) Perform the training and testing for each cycle.

(7) If the network produce acceptable results for all cycles, perform step 8 else perform step 5 to try other appropriate network structures else perform step 4 to try with other learning algorithm else perform step 3 to add or remove from input set.

Otherwise, go back to step 2 to try different neural network architecture.

(8) Finish - record the results

Fig 2: Algorithm for ANN predictive model

For our hybridized approach we identified 18 input variables to train the network comprising both technical variables and fundamental analysis variables.

\section{The technical analysis variables are:}

$\mathrm{O}_{i-1}$ the opening price of day $i-1$

$\mathrm{O}_{i-2}$ the opening price of day $i-2$

$\mathrm{H}_{i-1}$ the daily high price of day $i-1$

$\mathrm{H}_{i-2}$ the daily high price of day $i-2$

$\mathrm{L}_{i-1}$ the daily low price of day $i-1$

$\mathrm{L}_{i-2}$ the daily low price of day $i-2$

$\mathrm{C}_{i-1}$ the closing price of day $i-1$

$\mathrm{C}_{i-1}$ the closing price of day $i-2$

$\mathrm{V}_{i-1}$ the trading volume of day $i-1$

$\mathrm{V}_{i-2}$ the trading volume of day $i-2$

For the implementation of our hybridized approach, we experimented with the following different neural network model configurations 18-3-1, 18-4-1, 18-7-1, 18-9-1, 18-17-1, 18-21-1, 18-23-1, 18-25-1, 18-711 using the MATLAB Neural Network Tools Box version 7. 18 represent the number of input variables, the variable values in the middle of the configuration are the number of hidden neurons, and 1 depicts the expected single output of the ANN. Training data and testing data were carefully selected and we observed the various outcomes of the different network structure models implemented with MATLAB Neural Network Tools Box version 7. In training our network model, the test data is not used. It was trained for 10,000 epochs for each training set. The output of neural network model was analyzed by comparing the predicted values with the actual values over a sample period. For our output to be considered useful for trading decision support, overall hit rate of level of accuracy should be considerably high enough to be acceptable. The empirical results are presented in the next section.

\section{IV.}

\section{RESULTS AND DISCUSSION}

After several experiments with different network architectures, the network predictive model that gave the most accurate daily stock price prediction was 18-25-1 backpropagation network (BPN) using the hybridized approach that combines the variables of technical and fundamental analysis, were used. The results presented in table 1 were the findings from testing period (out of sample test data) over different network structures when a hybridized approach was used. Figure 3-11 illustrates the correlation of the level accuracy among different network structure with the hybridized approach by comparing the actual stock prices with the predicted values of stock prices. Over time, the network structure 18-25-1 (Fig.3) gave impressive results over different data set of different sample periods. We found out that the accuracy level of the hybridized approach is better than the technical analysis approach. Similarly, by the comparing the other figures in hybridized approach with the technical analysis approach. The prediction accuracy of hybridized approach was better off. Hence, the 
hybridized approach can be used successfully as decision-support in real-life trading in a way that will enhance the profiting of investors or traders on daily trading. The level of accuracy of the different ANN configurations is presented in the table 1 .

Table 1: Sample of Empirical Results of using Hybrid Approach on different Neural Network Predictive Models

\begin{tabular}{|c|c|c|c|c|c|c|c|c|c|c|c|}
\hline \multicolumn{12}{|c|}{ Sample of Daily Stock Price prediction } \\
\hline & \multirow{2}{*}{$\begin{array}{c}\text { Sample } \\
\text { Period }\end{array}$} & \multirow{2}{*}{$\begin{array}{l}\text { Actual } \\
\text { Value }\end{array}$} & \multicolumn{9}{|c|}{ Predicted Values with Different Neural Network Predictive Models } \\
\hline & & & $18-3-1$ & $18-4-1$ & $18-7-1$ & $18-9-1$ & $18-17-1$ & $18-21-1$ & $18-23-1$ & $18-25-1$ & $18-71-1$ \\
\hline 1 & $12 / 01 / 2013$ & 20.13 & 21.55 & 22.19 & 18.75 & 18.77 & 19.21 & 22.18 & 22.65 & 20.63 & 20.42 \\
\hline 2 & $12 / 02 / 2013$ & 19.26 & 18.10 & 18.20 & 22.43 & 18.00 & 17.95 & 17.24 & 16.97 & 19.31 & 17.14 \\
\hline 3 & $12 / 03 / 2013$ & & & & 21.07 & 21.03 & & & 19.40 & & 00 \\
\hline 4 & $12 / 04 / 2013$ & 21.09 & 19.34 & 21.73 & 19.33 & 19.35 & 19.14 & 23.08 & 23.75 & 22 & 21.87 \\
\hline 5 & $12 / 05 / 2013$ & 35 & 20.04 & 20.31 & 21.56 & 20.21 & .57 & 19.63 & 19.42 & 04 & 20.09 \\
\hline 6 & $12 / 08 / 2013$ & 19.84 & 22.76 & 17. & 22.74 & 21.66 & 23.08 & 28 & 18.98 & 13 & 21.45 \\
\hline 7 & $12 / 09 / 2013$ & 17.13 & 21.19 & 19.40 & 19.20 & 20.11 & 17.25 & 19.87 & 21.45 & 55 & 18.33 \\
\hline 8 & $12 / 10 / 2013$ & 18.96 & 18.34 & 15.96 & 21.39 & 21.08 & 24 & 22.54 & 21.47 & & 18.01 \\
\hline 9 & $12 / 11 / 2013$ & 20.51 & 22.09 & 20.71 & 17.34 & 19.25 & 18.37 & 20.94 & 16.73 & 20.98 & 21.14 \\
\hline 10 & $12 / 12 / 2013$ & & 19.00 & 20.96 & 16.99 & 23.49 & 20.14 & 22.60 & 18.72 & & 18.62 \\
\hline 11 & $12 / 15 / 2013$ & 19.55 & 17.58 & & 20.47 & 18.21 & 19.40 & 21.76 & 23.04 & 89 & 19.20 \\
\hline 12 & $12 / 16 / 2013$ & 19.67 & 17.43 & 19.00 & 22.16 & 16.12 & 19.34 & 18.20 & 21.79 & 20.41 & 22.83 \\
\hline 13 & $12 / 17 / 2013$ & 20.32 & 21.97 & 22.92 & 19.01 & 23.17 & 19.25 & 19.41 & 19.68 & 20.33 & 18.14 \\
\hline 14 & $12 / 18 / 2013$ & 21.88 & 22.67 & 18.58 & 19.32 & 21.68 & 21.57 & 19.00 & 18.84 & 21.90 & 22.12 \\
\hline
\end{tabular}
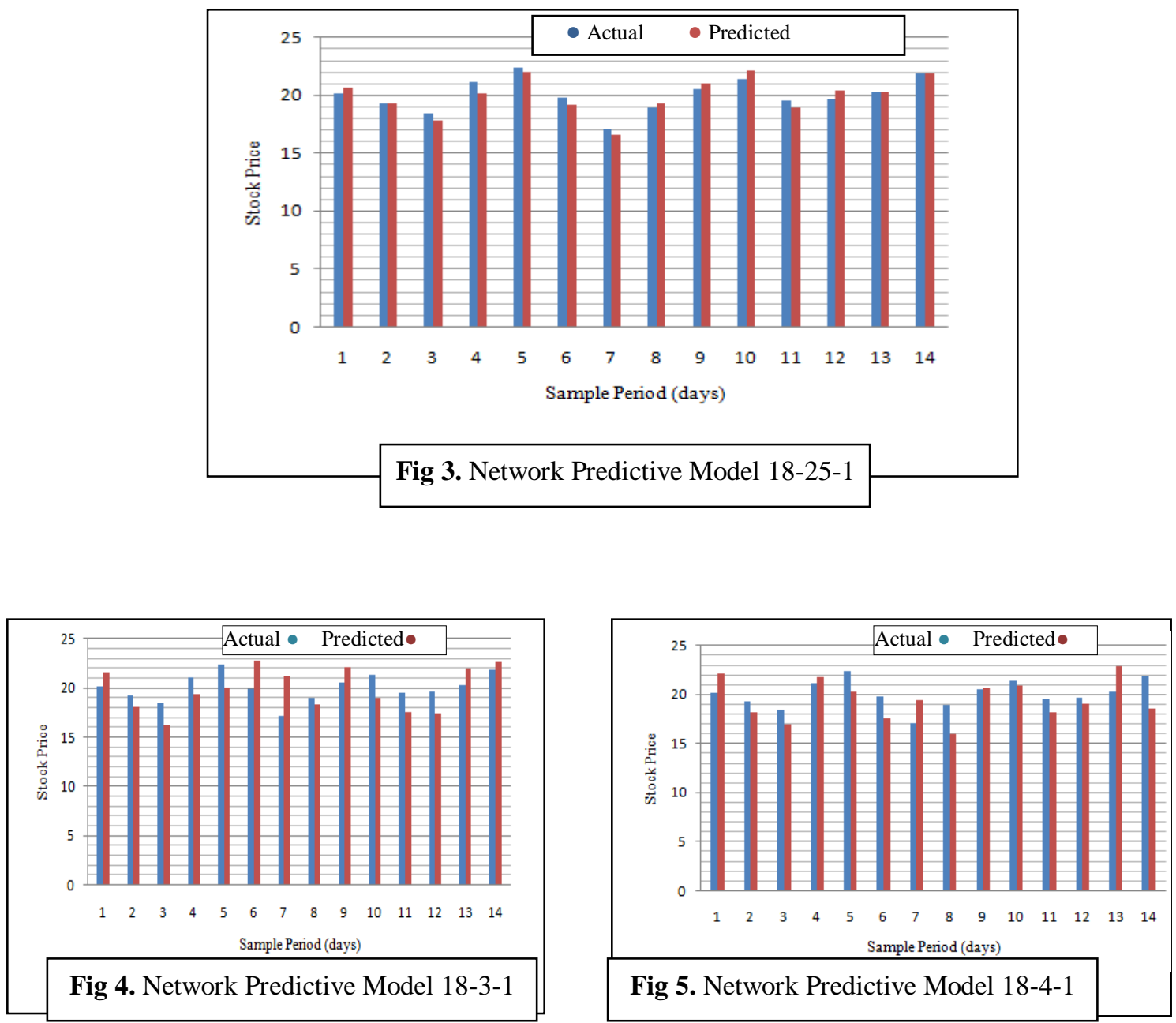


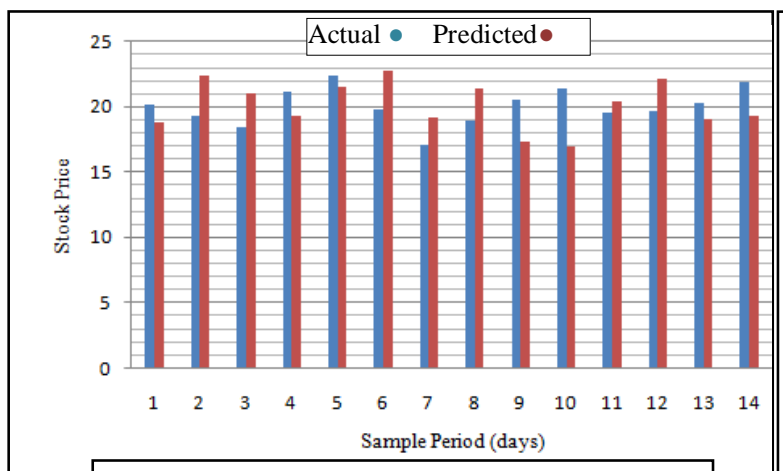

Fig 6. Network Predictive Model 18-7-1

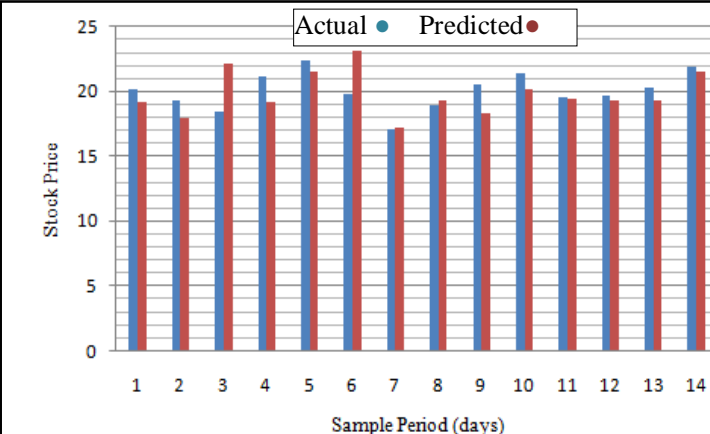

Fig 8. Network Predictive Model 18-17-1

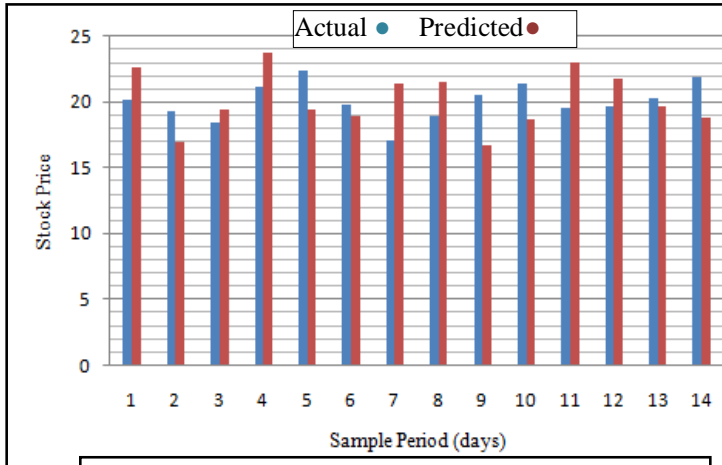

Fig 10. Network Predictive Model 18-23-1



Fig 7. Network Predictive Model 18-9-1

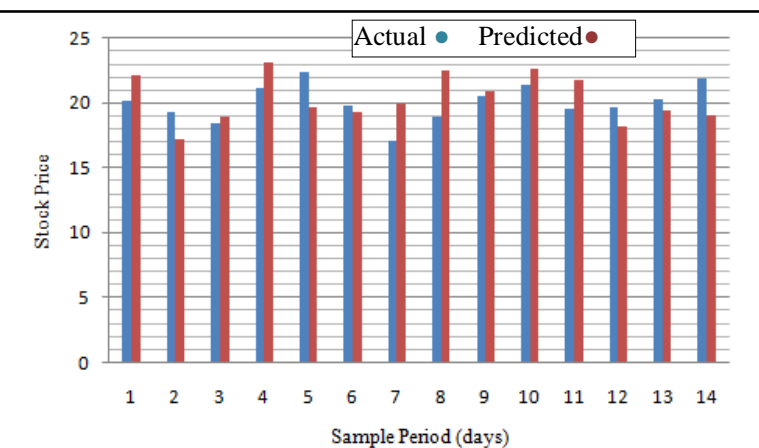

Fig 9. Network Predictive Model 18-21-1



Fig 11. Network Predictive Model 18-71-1

\section{CONCLUSION}

This paper reported a hybridized approach of the combination of the variables of technical analysis and fundamental analysis to design and implementation a feed forward multilayer perceptron ANN predictive model trained with a backprogation algorithm for improved accuracy of stock prediction and the evaluation of the usefulness of the ANNs for use as decision support in a real trading situation. The characteristics of the suggested model include symbolizing of financial time series, which reduces its volatility without losing data quality as in the case of smoothing.

To determine the performance of our model, an empirical study was carried out with the published stock data obtained from the Internet, where the hybridized approach was compared with the use of only technical analysis. The empirical results obtained showed high level of accuracy for daily stock price prediction with a hybridized approach performing better than the technical analysis approach. Therefore, the hybridized approach has the potential to enhance the quality of decision making of investors in the stock market by offering more accurate 
stock prediction compared to existing technical analysis based approach. In future work, we intend to determine the critical impact of specific fundamental analysis variables on quality of stock price prediction.

\section{REFERENCES}

[1] M.T. Philip, K. Paul, S.O. Choy, K. Reggie, S.C. Ng, J. Mak, T. Jonathan, K. Kai, and W. TakLam, "Design and Implementation of NN5 for Hong Stock Price Forecasting", Journal of Engineering Applications of Artificial Intelligence, vol. 20, 2007, pp. 453-461.

[2] J. Yao, C. Tan and H. Poh, "Neural Networks for Technical Analysis: A Study on KLCI," International Journal of Theoretical and Applied Finance, Vol. 2, No. 2, 1999, pp. 221-241. doi: 10.1142/S0219024999000145

[3] K. H. Lee and G. S. Jo, "Expert System for Predicting Stock Market Timing Using a Candlestick Chart," Expert Systems with Applications, Vol. 16, No. 4, 1999, pp. 357- 364. doi:10.1016/S0957-4174(99)0011-1

[4] A. S. Chen, M. Leung and H. Daouk, "Application of Neural Networks to an Emerging Financial Market: Forecasting and Trading the Taiwan Stock Index," Computers \& Operations Research, Vol. 30, No. 6, 2003, pp. 901-923. doi:10.1016/S0305-0548 (02)00037-0

[5] I. Kaastra and M. Boyd, "Designing a Neural Network for Forecasting Financial and Economic Time Series," Neurocomputing, Vol. 10, No. 3, 1996, pp. 215-236. doi:10.1016/0925-2312 (95) 00039-9

[6] E.F.Fama,The behavior of Stock market prices,J.Bus.38 (1965)34-105.

[7] Ying Han, Emilio Corchado, Colin Fyfe, Forecasting using twinned principal curves and twinned selforganizing maps, Neurocomputing 57 (2004), 37-47.

[8] V. Sakalauskas, D. Kriksciuniene, Analysis of theday-of-the-weekeffectin emerging stockmarkets. Lecture Notesin Artificial Intelligence, 4874, Springer Verlag, Berlin, Heidelberg, 2007,371-380.

[9] Leandro S. Neural Networks Applied To Stock Market Forecasting: An Empirical Analysis, Journal of the Brazilian Neural Network Society, Vol. 8, 3-22, 2010.

[10] HUANG, W., LAI, K. K., NAKAMORI, Y., WANG, S. Y., and YU, L. Neural networks in finance and economics forecasting. International Journal of Information Technology and Decision Making, 6, 113$140,2007$.

[11] LO, A. W., and MACKINLAY, A. C. Stock market prices do not follow random walks: Evidence from a simple specification test. Review of Financial Studies, 1, 41-66, 2008.

[12] Ghezelbash. Ali. Predicting Changes in Stock Index and Gold Prices to Neural Network Approach, The Journal of Mathematics and Computer Science Vol. 4 No.2, pp. 227 - 236, 2012.

[13] Hagan. M. "Neural Network Design". PWS Publ. Company. 1996

[14] Haykin, S. 1998. Neural Networks: a Comprehensive Foundation, seconded. Prentice-Hall, Englewood Cliffs, NJ.

[15] Rumelhart, D.E., Hinton, G.E., Williams, R.J., 1986b. Learning internal representations by backpropagating errors. In: Rumelhart, D.E., McClelland, J.L., and the PDP Research Group, (Eds.), Parallel Distributed Processing, vol. 1, MIT Press, Cambridge, MA, pp. 318-362 (Chapter 8).

[16] Y.F. Sun, Y.C. Liang, W.L. Zhang, H.P. Lee, W.Z. Lin, and L.J. Cao, "Optimal Partition Algorithm of the RBF Neural Network and Its Application to Financial Time Series Forecasting", Neural Computation \& Application, 2005, 14, pp. 36-44.

[17] D. Zhora, "Data Preprocessing for Stock Market Forecasting using Subspace Classifier Network", Proceedings of the International Joint Conference on Neural Networks, Montreal, 2005, pp. 2549-2554.

[18] Vapnik, V.N., 1995. The Nature of Statistical Learning Theory. Springer, Berlin. 